\title{
Na-based crystal scintillators for next-generation rare event searches
}

\author{
S. Nagorny ${ }^{\mathrm{a}}$, C. Rusconi ${ }^{\mathrm{b}, \mathrm{c}, *}$, S. Sorbino ${ }^{\mathrm{d}, \mathrm{e}}$, J.W. Beeman ${ }^{\mathrm{f}}$, F. Bellini ${ }^{\mathrm{d}, \mathrm{e}}$, L. Cardani ${ }^{\mathrm{e}}$, V.D. Grigorieva ${ }^{\mathrm{g}}$, L. Pagnanini ${ }^{\text {h,i }}$, S. Nisi ${ }^{\mathrm{c}}$, \\ S. Pirro ${ }^{\mathrm{c}}$, K. Schäffner ${ }^{\mathrm{j}}$, V.N. Shlegel ${ }^{\mathrm{g}, \mathrm{k}}$
}

\author{
${ }^{a}$ Queen's University, Physics Department, K7L 3N6, Kingston (ON), Canada \\ ${ }^{b}$ Department of Physics and Astronomy, University of South Carolina, Columbia, SC 29208 - USA \\ ${ }^{c}$ INFN - Laboratori Nazionali del Gran Sasso, 67010 Assergi - Italy \\ ${ }^{d}$ Dipartimento di Fisica, Sapienza Università di Roma, P.le Aldo Moro 2, 00185, Rome, Italy \\ ${ }^{e}$ INFN - Sezione di Roma 1, 00185 Roma - Italy \\ ${ }^{f}$ Lawrence Berkeley National Laboratory, Berkeley, California 94720, USA \\ ${ }^{g}$ Nikolaev Institute of Inorganic Chemistry, 630090 Novosibirsk - Russia \\ ${ }^{h}$ Dipartimento di Fisica, Università di Milano - Bicocca, 20126 Milano - Italy \\ ${ }^{i}$ INFN - Sezione di Milano Bicocca, 20126 Milano - Italy \\ ${ }^{j}$ Max-Planck-Institut für Physik, 80805 München - Germany \\ ${ }^{k}$ Novosibirsk State Tech University, 20 Karl Marx Prospect, 630092 Novosibirsk - Russia
}

\section{Abstract}

The growing interest in clarifying the controversial situation in the Dark Matter sector has driven the experimental efforts towards new ways to investigate the long-standing DAMA/LIBRA result. Among them, low-temperature calorimeters based on Na-containing scintillating crystals offer the possibility to clarify the nature of the measured signal via particle identification. Here we report the first measurement of Na-containing crystals, based on material different from $\mathrm{NaI}_{\text {, i.e. }} \mathrm{Na}_{2} \mathrm{Mo}_{2} \mathrm{O}_{7}$ and $\mathrm{Na}_{2} \mathrm{~W}_{2} \mathrm{O}_{7}$, pointing out their excellent performance in term of energy resolution, light yield, and particle identification.

Keywords: Double Beta Decay, Dark Matter, Scintillating bolometers, Particle identification methods, Cryogenic Detectors PACS: 07.20.Mc, 07.57K.Kp, 23.40.-s, 29.40Ka, 29.40.Mc

\section{Introduction}

Several astronomical observations have shown that the visible and ordinary matter represents only a small fraction of the Universe, a large part of which is expected to be in the form of non-visible components, namely Dark Matter (DM), and Dark Energy [1].

- While the Cosmology seems to corroborate this hypothesis, direct DM searches depict a controversial scenario. The DAMA/LIBRA collaboration, operating $250 \mathrm{~kg}$ of $\mathrm{NaI}(\mathrm{Tl})$ scintillators, measures a signal compatible with the presence of DM in our galactic halo [2]. The other experiments in this sector, even if their sensitivity is such as to measure the DAMA/LIBRA signal, do not observe it.

This comparison among experiments based on different target materials, relies on the so-called standard assumptions about the DM halo distribution and the DM-nucleus crosssection, whose dependence on the atomic mass and the nuclear form factor is unknown. The first step to solve the puzzle is a model-independent cross-check of the DAMA/LIBRA signal, which is feasible only using the same target material.

I-based experiments (KIMS [3], PICO [4]) have already ruled out the participation of iodine in the measured DAMA/LIBRA signal, while NaI-based experiments are currently in operation (ANAIS [5], COSINE [6]) or construction

\footnotetext{
* Corresponding author

Email address: Claudia. Rusconi@LNGS. INFN . IT (C. Rusconi)
}

(SABRE [7]). These experiments exploit the same technique as DAMA/LIBRA, as they measure the scintillation light produced by particle interactions in $\mathrm{NaI}(\mathrm{Tl})$ crystals.

Recently, the COSINUS collaboration has demonstrated that $\mathrm{NaI}$ and CsI crystals can also be operated as low-temperature calorimeters [8, 9]. Taking advantage from the lower energy threshold, and the particle identification capability [10], lowtemperature calorimeters have promising prospects to give new insights on the long-standing DAMA/LIBRA claim.

The same technique can also be used with other Nacontaining crystals, in order to overcome some technical difficulties related to the hygroscopicity of NaI.

In this paper we investigate for the first time two Nacontaining crystals, $\mathrm{Na}_{2} \mathrm{Mo}_{2} \mathrm{O}_{7}$ and $\mathrm{Na}_{2} \mathrm{~W}_{2} \mathrm{O}_{7}$, and prove that they can be operated as low-temperature calorimeters obtaining excellent performance term of energy resolution, light yield, and particle discrimination.

\section{Cryogenic calorimeters}

Cryogenic calorimeters, historically also called bolometers, are one of the most promising technologies for the search of rare events [11] such as neutrinoless double beta decay (CUORE [12], CUPID-0 [13, 14, 15], CUPID-Mo [16, 17], AMoRE [18]) and dark matter interactions (CRESST [19], COSINUS [8], EDELWEISS [20], SuperCDMS [21]). 
The working principle of these devices is rather simple: energy deposits into an absorber give rise to temperature variations, which can be converted into electrical signals through dedicated sensors.

Common absorbers are dielectric and diamagnetic crystals, for which we can assume a lattice-dominated specific heat:

$$
c(T)=\frac{12}{5} \pi^{4} N_{A} k_{B}\left(\frac{T}{\Theta_{D}}\right)^{3}
$$

where $\mathrm{N}_{A}$ is the Avogadro Number, $\mathrm{k}_{B}$ the Boltzmann constant and $\Theta_{D}$ the Debye temperature.

If the crystal is cooled at cryogenic temperatures $(\approx 10 \mathrm{mK})$ the thermal capacitance $C$ can reach values as low as $10^{-9}$ $10^{-10} \mathrm{~J} / \mathrm{K}$ (depending on the exact values of $\Theta_{D}$ and of the crystal mass). Such a small capacitance allows to measure temperature variation up to a few hundreds of $\mu \mathrm{K}$ for an energy deposit of $1 \mathrm{MeV}$ in the crystal.

The temperature variations are then converted into sizable voltage signals exploiting dedicated sensors. In this work, we used Neutron Transmutation Doped Germanium thermistors (NTD) attached to the crystals. Typical voltage signals produced by NTDs ranges from hundreds to thousands of $\mu \mathrm{V} / \mathrm{MeV}$. The light detectors (LD) used with this technique are themselves bolometers [22]: they usually consists of a thin semiconductor (dark) crystal wafer that absorbs the scintillation photons giving rise to a sizable heat pulse that is proportional to the number of absorbed photons.

The technique of cryogenic calorimeters offers an exquisite energy resolution (usually around $0.1 \%$ ) and versatility in the choice of the compounds, enabling the investigation of different materials. Nevertheless, the crystals operated as absorbers must feature good phonons propagation and excellent radiopurity. Moreover, crystals emitting a large amount of light at cryogenic temperatures are preferable, as they allow the simultaneous measurement of the heat signal and of the emitted light, enabling particle identification.

\section{Crystal production}

The crystals characterized in this work were produced at Nikolaev Institute of Inorganic Chemistry, Siberian Branch, Russian Academy of Science, at Novosibirsk, Russia, using the Low Temperature Gradient Czochralski (LTG-Cz) technique operating three-zone furnace [23, 24, 25].

The distinctive feature of this method is a temperature gradient of $0.11 .0 \mathrm{~K} / \mathrm{cm}$ in the melt, much lower in comparison to the conventional Czochralski technique in which the temperature gradient is around $10100 \mathrm{~K} / \mathrm{cm}$ (see [26] for more details). The second important feature of this technique is the use of closed platinum crucibles. Combination of these two features leads to the minimization of charge components evaporation from the melt, and losses of the initial materials do not exceed $0.5 \%$.

Standard market $4 \mathrm{~N}$ purity grade $\mathrm{Na}_{2} \mathrm{CO}_{3}$ and $\mathrm{WO}_{3}$ powders were used as starting materials for the growth of $\mathrm{Na}_{2} \mathrm{~W}_{2} \mathrm{O}_{7}$ as described in details in [27].

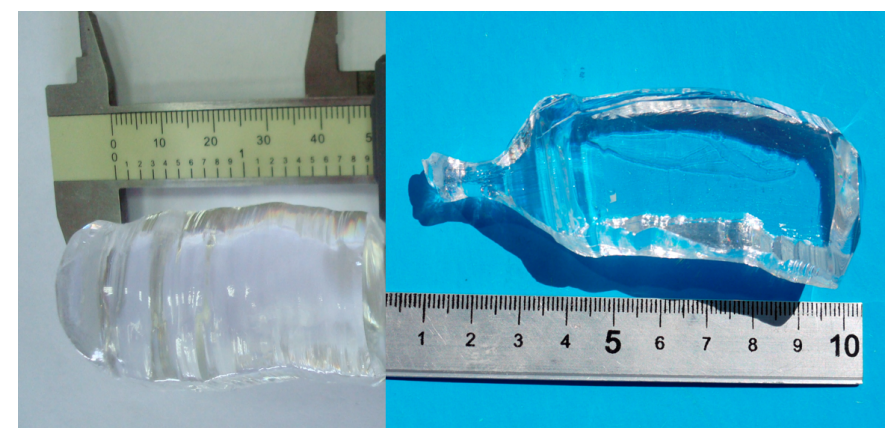

Figure 1: Picture of the $\mathrm{Na}_{2} \mathrm{~W}_{2} \mathrm{O}_{7}$ (left) and $\mathrm{Na}_{2} \mathrm{Mo}_{2} \mathrm{O}_{7}$ (right) crystalline boules.

The stoichiometric mix of $\mathrm{Na}_{2} \mathrm{CO}_{3}$ and $\mathrm{WO}_{3}$ powders was used as starting materials for the charge preparation: they were allowed to react at $650{ }^{\circ} \mathrm{C}$ for $12 \mathrm{~h}$ into the platinum crucible according to the reaction: $\mathrm{Na}_{2} \mathrm{CO}_{3}+2 \mathrm{WO}_{3} \rightarrow \mathrm{Na}_{2} \mathrm{~W}_{2} \mathrm{O}_{7}+$ $\mathrm{CO}_{2} \uparrow$. The obtained $\mathrm{Na}_{2} \mathrm{~W}_{2} \mathrm{O}_{7}$ powder was then further annealed at $750{ }^{\circ} \mathrm{C}$ for 12 hours in the same crucible and in air atmosphere to yield the final charge. Finally the crystal was pulled along [001] axis at $1.52 .5 \mathrm{~mm} / \mathrm{h}$ rate and rotation rate $312 \mathrm{rpm}$, respectively. A colorless, transparent and nonhygroscopic $\mathrm{Na}_{2} \mathrm{~W}_{2} \mathrm{O}_{7}$ single crystal with excellent phase purity was obtained [27] as shown in Fig. 1](left).

Deeply purified molybdenum oxide $\left(\mathrm{MoO}_{3}\right)$ [28, 29] and commercial sodium carbonate $\mathrm{Na}_{2} \mathrm{CO}_{3}$ (4N purity grade) were used for the solid-state synthesis of $\mathrm{Na}_{2} \mathrm{Mo}_{2} \mathrm{O}_{7}$ powder according to the reaction: $2 \mathrm{MoO}_{3}+\mathrm{Na}_{2} \mathrm{CO}_{3} \rightarrow \mathrm{Na}_{2} \mathrm{Mo}_{2} \mathrm{O}_{7}+\mathrm{CO}_{2} \uparrow$. The synthesis was carried out in a platinum crucible with dimensions $70 \mathrm{~mm}$ (dia) and $130 \mathrm{~mm}$ (length). The compound was heated up to $350{ }^{\circ} \mathrm{C}$ with the rate of $100{ }^{\circ} \mathrm{C} / \mathrm{h}$, then heated up to $650{ }^{\circ} \mathrm{C}$ with the rate $20^{\circ} \mathrm{C} / \mathrm{h}$ and held at this temperature for $5 \mathrm{~h}$ for homogenization of the melt. The $\mathrm{Na}_{2} \mathrm{Mo}_{2} \mathrm{O}_{7}$ crystal was then grown in the same crucible and pulled along [010] axis with $0.32 .5 \mathrm{~mm} / \mathrm{h}$ rate and rotation rate $312 \mathrm{rpm}$, respectively. A high optical quality, colorless $\mathrm{Na}_{2} \mathrm{Mo}_{2} \mathrm{O}_{7}$ single crystal was obtained [30] and is presented in Fig. 1](right).

Both crystals, disodium molybdate and disodium tungstate, were grown in air atmosphere.

Finally, two $10 \times 10 \times 10 \mathrm{~mm}^{3}$ samples of $\mathrm{Na}_{2} \mathrm{Mo}_{2} \mathrm{O}_{7}$ and $\mathrm{Na}_{2} \mathrm{~W}_{2} \mathrm{O}_{7}$ crystals were cut from the single crystalline boules for characterization as scintillating bolometers. A sample of each crystal was then tested for internal contamination using a High Resolution Inductively Coupled Plasma-Mass Spectrometer (HR-ICP-MS, Thermo Fisher Scientific ELEMENT2) at the Laboratori Nazionali del Gran Sasso of INFN (LNGS, Italy). The results of the analyses are listed in Tab. 1 .

\section{Experimental setup}

The mounting hosting the crystals consists of a copper basement covered with a sheet of a plastic reflector (Vikuiti ${ }^{T M}$ ) on which the crystals rest without any holding structure. Moreover, the plastic reflector tightly surrounds the lateral faces of each 


\begin{tabular}{lcc} 
Element & $\mathrm{Na}_{2} \mathrm{Mo}_{2} \mathrm{O}_{7}$ & $\mathrm{Na}_{2} \mathrm{~W}_{2} \mathrm{O}_{7}$ \\
\hline \hline$K$ & $<15$ & $<10$ \\
$C r$ & $<.02$ & $<.02$ \\
$M n$ & $<.03$ & $<.03$ \\
$F e$ & $<5$ & $<5$ \\
$\mathrm{Ni}$ & $<.05$ & $<.05$ \\
$\mathrm{Zn}$ & $<.02$ & $<.02$ \\
$\mathrm{Cd}$ & $<20$ & $\mathbf{2 . 5}$ \\
$\mathrm{Sn}$ & $<2$ & $<2$ \\
$\mathrm{Sb}$ & $<.02$ & $<.02$ \\
$\mathrm{~Pb}$ & $\mathbf{. 0 3}$ & $\mathbf{. 0 2}$ \\
$\mathrm{Th}$ & $<.02$ & $<.1$ \\
$U$ & $<.02$ & $<.03$ \\
\hline \hline
\end{tabular}

Table 1: Contaminations in ppm obtained with ICPMS on some common metals. Samples were dissolved in a 1:5 solution of $\mathrm{HF}$ and $\mathrm{HNO}_{3}$ at $75^{\circ} \mathrm{C}$ with dilution factors of $470\left(\mathrm{Na}_{2} \mathrm{Mo}_{2} \mathrm{O}_{7}\right)$ and $680\left(\mathrm{Na}_{2} \mathrm{~W}_{2} \mathrm{O}_{7}\right)$.

crystal, to maximize the light collection while keeping them in position.

Each crystal is equipped with a $2.85 \times 2 \times 0.5 \mathrm{~mm}^{3} \mathrm{NTD}$ through six dots of Araldit Rapid ${ }^{T M}$ glue $(\sim 0.05 \mathrm{~mm}$ height, $\sim 0.7 \mathrm{~mm}$ diameter). The NTD thermistors are provided with $50 \mu \mathrm{m}$ thick gold wires for the sensor readout, which are connected to electrically insulated copper pins held in a copper frame surrounding the mounting. The pins are then connected to the cryostat readout through copper wires.

On the top of the copper frame surrounding the crystals, a second frame is placed, hosting the light detector (LD). It consists of a Ge wafer ( $50.8 \mathrm{~mm}$ diameter, $0.2 \mathrm{~mm}$ thick), equipped with an NTD thermistor (size $3 \times 1 \times 0.5 \mathrm{~mm}^{3}$ ) and held in the copper frame through two PTFE clamps. The detector is showed in (Fig. 2).

Finally, in order to avoid vibrations reaching the detectors, the setup is mechanically decoupled from the cryostat by utilizing a two-stage pendulum system [22] and cooled down to about $10 \mathrm{mK}$ in the CUPID R\&D cryostat, a ${ }^{3} \mathrm{He} /{ }^{4} \mathrm{He}$ dilution refrigerator installed deep underground in Hall $\mathrm{C}$ of the Laboratori Nazionali del Gran Sasso, Italy.

Two sources are included inside the mounting for the detector calibration, a ${ }^{55} \mathrm{Fe}$ spot facing the LD, and a Thoriated Tungsten wire $(1 \% \mathrm{Th})$ lying between the crystals.

The NTD thermistors are biased with a steady current through large $(27+27$ or $2+2 \mathrm{G} \Omega)$ load resistors [31]. When the thermistor resistance changes because of an energy release in its absorber, a voltage variation happens across the currentbiased thermistor, which is amplified by the front-end electronics located just outside the cryostat [32].

The signals are then filtered by a 6-pole low pass BesselThomson filter (roll-off rate of $120 \mathrm{~dB} /$ decade) with a cut-off frequency of $63 \mathrm{~Hz}(200 \mathrm{~Hz}$ for the LD), and fed into an 18 bit NI-6284 PXI ADC unit. The sampling frequency is $1 \mathrm{kHz}$ for all the channels, while the length of the acquisition window is optimized for each detector according to the different timedevelopment of the signals in each crystal, i.e., $\mathrm{Na}_{2} \mathrm{~W}_{2} \mathrm{O}_{7}$ (1 $\mathrm{s}), \mathrm{Na}_{2} \mathrm{Mo}_{2} \mathrm{O}_{7}(500 \mathrm{~ms})$, and $\mathrm{LD}(500 \mathrm{~ms})$. When a trigger

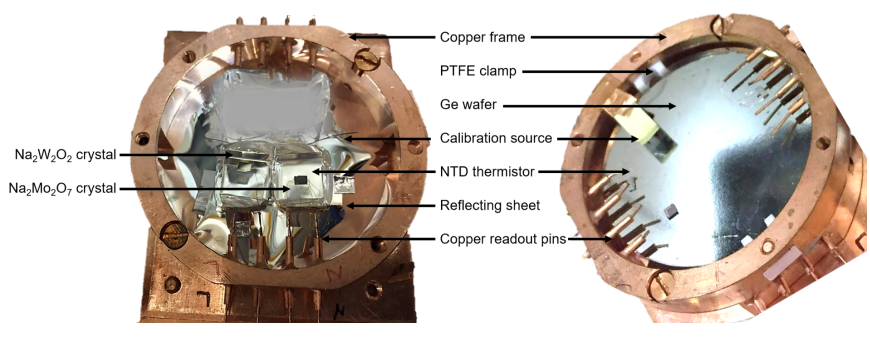

Figure 2: Picture of the experimental setup, before (left) and after (right) placing the light detector on the Na containing crystals.

fires on a detector, a waveform is acquired and saved on disk for the off-line analysis. Moreover, when a trigger fires for a Na-containing crystal, the corresponding waveform of the LD is also acquired, regardless of its trigger. More details about the DAQ system can be found in [33].

\section{Performance and results}

\subsection{Data processing}

Each triggered waveform is analyzed off-line to extract the signal amplitude and other important parameters to quantify the detector performance. To improve the signal-to-noise ratio, we apply the optimum filter [34], a software algorithm that suppresses the frequency components of the signal most affected by noise. The construction of the transfer function $\mathrm{H}(\omega)$ of this filter requires the knowledge of the noise power spectra $\mathrm{N}(\omega)$, and the ideal response $S(t)$ of each detector:

$$
H(\omega)=k \frac{S^{*}(\omega)}{N(\omega)} e^{i \omega t_{M}}
$$

where $S^{*}(\omega)$ is the Fourier transform of $S(t)$ and $t_{M}$ is the time at which the filtered signal is maximum. $S(t)$ is obtained by averaging hundreds of high energy signals, in order to suppress the contributions due to stochastic noise. To construct the noise power spectra $\mathrm{N}(\omega)$, we randomly acquire noise windows (i.e. with no pulses therein), and average the square module of their discrete Fourier transform in the frequency domain.

Each pulse is Fourier transformed and filtered using $\mathrm{H}(\omega)$ to obtain a more precise evaluation of its amplitude. The optimum filter allows to extract also some parameters related to the signal time-development, such as the rise time and decay time, defined as the time difference between the $90 \%$ and the $10 \%$ of the rising edge, and the time difference between the $30 \%$ and $90 \%$ of the trailing edge, respectively.

Since the light pulses show a worse signal-to-noise ratio compared to the heat pulses, we estimate their amplitude by accounting for the (known) time-delay between heat and corresponding light signal. More details about this algorithm can be found in [35]. The amplitude extracted by the optimum filter is corrected against temperature drifts by exploiting the physics peak with at the highest energy (the $2.6 \mathrm{MeV}$ line for $\mathrm{Na}_{2} \mathrm{~W}_{2} \mathrm{O}_{7}$ and the $238 \mathrm{keV}$ line for $\mathrm{Na}_{2} \mathrm{Mo}_{2} \mathrm{O}_{7}$ ). 
Finally, the corrected amplitude is converted into energy. The scintillating crystals are calibrated using the known peaks produced by the thoriated tungsten wire that permanently illuminated the devices. Since the large majority of the energy is released in the form of heat, we expect the light signals to be much smaller (from a few $\mathrm{keV}$ to tens of $\mathrm{keV}$ depending on the light output of the crystal). Thus, the light detector is calibrated using the 5.9 and $6.4 \mathrm{keV}$ peaks produced by the ${ }^{55} \mathrm{Fe} \mathrm{X}$-rays source placed on top of the Ge crystal (see Fig. 2 right).

The general features of the detectors are summarized in Tab.2.

\begin{tabular}{lcccc} 
& $\begin{array}{c}A_{S} \\
{[\mu \mathrm{V} / \mathrm{MeV}]}\end{array}$ & $\begin{array}{c}\sigma_{\text {baseline }} \\
{[\mathrm{keV} \text { RMS }]}\end{array}$ & $\begin{array}{c}\tau_{r} \\
{[\mathrm{~ms}]}\end{array}$ & $\begin{array}{c}\tau_{d} \\
{[\mathrm{~ms}]}\end{array}$ \\
\hline \hline $\mathrm{Na}_{2} \mathrm{Mo}_{2} \mathrm{O}_{7}$ & 231 & 0.2 & 9.1 & 30 \\
\hline $\mathrm{Na}_{2} \mathrm{~W}_{2} \mathrm{O}_{7}$ & 842 & 0.1 & 7.5 & 22 \\
\hline $\mathrm{Ge}-\mathrm{LD}$ & 1171 & 0.3 & 2.2 & 7.0 \\
\hline \hline
\end{tabular}

Table 2: Parameters of the cryogenic calorimeters. Voltage response of the thermistor for $1 \mathrm{MeV}$ energy deposit in the crystal (before amplification) $A_{S}$, energy resolution of the detector baseline after applying the optimum filter $\left(\sigma_{\text {baseline }}\right)$, rise $\left(\tau_{r}\right)$ and decay $\left(\tau_{d}\right)$ times of the pulses.

\subsection{Results from $\mathrm{Na}_{2} \mathrm{~W}_{2} \mathrm{O}_{7}$}

We report in Fig. 3 the energy spectrum obtained with the $1 \mathrm{~cm}^{3}$ (5.6. g) $\mathrm{Na}_{2} \mathrm{~W}_{2} \mathrm{O}_{7}$ crystal exposed to the thoriated tungsten wire.

The proximity of the $\gamma$-source to the $\mathrm{Na}_{2} \mathrm{~W}_{2} \mathrm{O}_{7}$ detector, as well as the presence of tungsten in the crystal (that increases the probability of photoelectric effect), allow to detect a large number of peaks, from $58 \mathrm{keV}$ (W X-ray escape) up to 2615 $\mathrm{keV}\left({ }^{208} \mathrm{Tl}\right)$. We fit each of the 20 detected peaks with a Gaussian function to derive the energy resolution in function of the energy, that increases linearly, as shown in Fig. 4 (right).

To energy-calibrate this detector, we use a fourth-degree polynomial function obtaining residuals smaller than $200 \mathrm{eV}$ in the whole considered energy range and better than $100 \mathrm{eV}$ for the vast majority of peaks (Fig. 4 (left)).

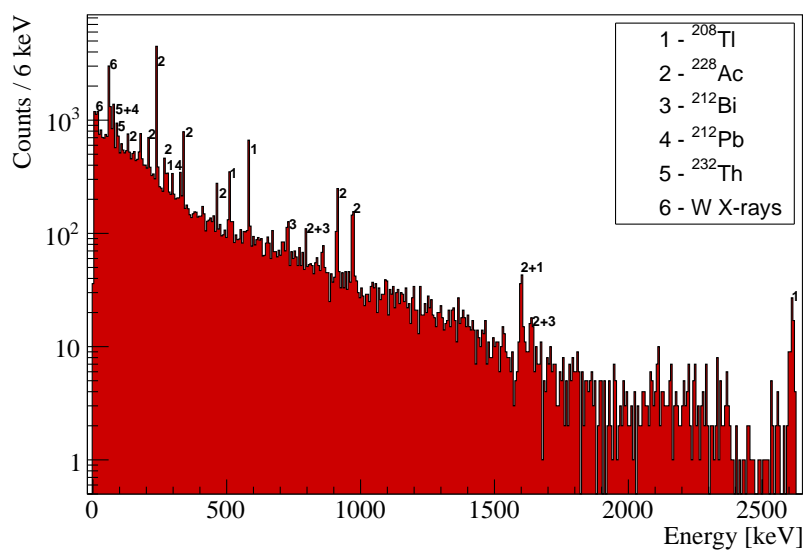

Figure 3: Energy spectrum of the $\mathrm{Na}_{2} \mathrm{~W}_{2} \mathrm{O}_{7}$ crystal exposed to the thoriated tungsten wire.
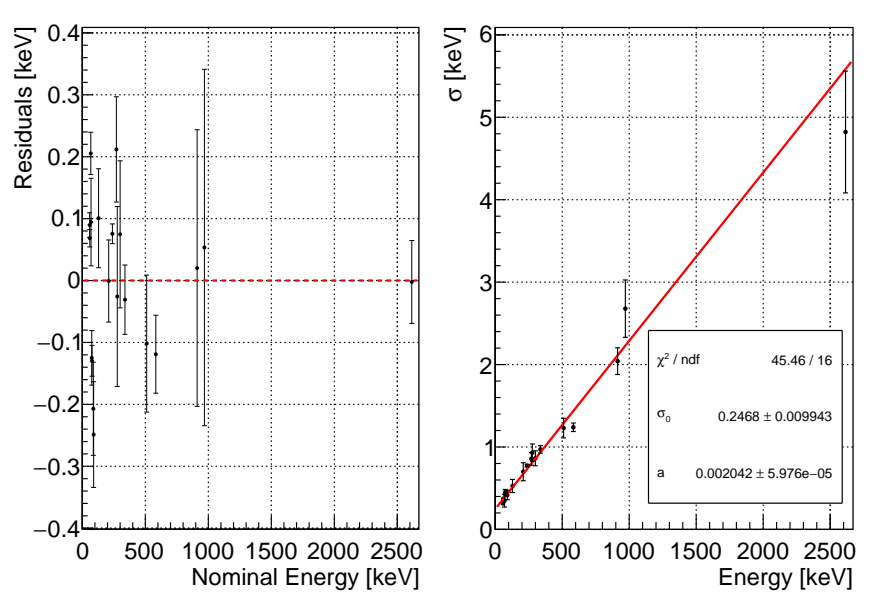

Figure 4: Left: Residuals of the energy calibration (nominal energy - reconstructed energy) as a function of the energy for $\mathrm{Na}_{2} \mathrm{~W}_{2} \mathrm{O}_{7}$. Right: energy resolution as a function of the energy. We modeled the dependency on the energy using a linear function $\sigma(E)=\sigma_{0}+a E$

We report in Fig. 5 the detected light as a function of the heat signal obtained with the use of a AmBe neutron source placed outside the cryostat. We can distinguish three populations, ascribed to $\beta / \gamma, \alpha$ 's and neutron events.

The light output of $\beta / \gamma$ events increases linearly with the energy and was evaluated with a linear fit up to $5 \mathrm{MeV}$ to include high energy $\gamma$ 's emitted during the run with the AmBe source.

The light yield (LY), defined as the measured light for a $\mathrm{MeV}$ energy deposit, results $\mathrm{LY}_{\beta / \gamma}=12.80 \pm 0.1 \mathrm{keV} / \mathrm{MeV}$.

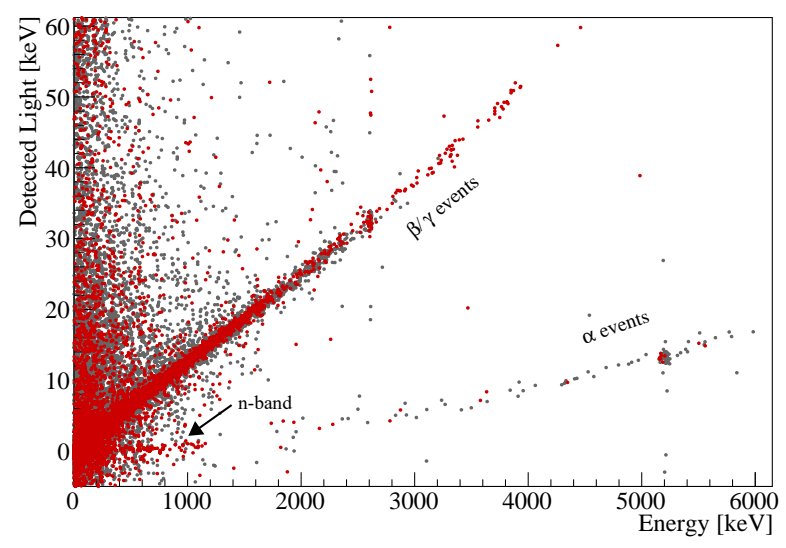

Figure 5: Detected light as a function of the heat measured with the $\mathrm{Na}_{2} \mathrm{~W}_{2} \mathrm{O}_{7}$ detector. Gray: run with the thoriated tungsten wire. Red: run with the thoriated tungsten wire and the AmBe neutron source. The nuclear recoils region extends up to $\approx 1 \mathrm{MeV}$ and is highlighted by the black arrow.

For a quick comparison with $\beta / \gamma$ events, we report the $\mathrm{LY}_{\alpha}$ measured using the only visible $\alpha$ peak, i.e. the $5304 \mathrm{keV}$ line of ${ }^{210} \mathrm{Po}, \mathrm{LY}_{\alpha}\left({ }^{210} \mathrm{Po}\right)=2.61 \pm 0.1 \mathrm{keV} / \mathrm{MeV}$. The light Quenching Factor (QF) of alphas (evaluated at $5.3 \mathrm{MeV}$ ) is therefore $0.204 \pm 0.008$, in good agreement with other crystal compounds [36].

Finally, we investigated the energy region of nuclear recoils, that appear when the detector is exposed to the neutron source. 
The energy resolution of the LD prevents an accurate analysis of the light emitted by these interactions, on which however we can put an upper limit of Light ${ }_{\text {nucl. }}<260 \mathrm{eV}$.

\subsection{Results from $\mathrm{Na}_{2} \mathrm{Mo}_{2} \mathrm{O}_{7}$}

Due to the lower density and smaller photoelectric crosssection, the $1 \mathrm{~cm}^{3}$ (3.6. g) $\mathrm{Na}_{2} \mathrm{Mo}_{2} \mathrm{O}_{7}$ crystal is less prone to show high energy lines, the largest one being the $\sim 239 \mathrm{keV}$ peak of ${ }^{212} \mathrm{~Pb}$ (Fig. 6).

We energy-calibrate the spectrum by applying the same procedure detailed before. The calibration is done with a third degree polynomial function and shows residuals smaller than 100 $\mathrm{eV}$ in the whole energy range of interest (Fig. 7(left)).

Also in this case, the energy resolution of the device, shown in Fig. 7(right), increases with the energy and can be modelled as $\sigma(E)=\sigma_{0}+a E$, with $\sigma_{0}=222 \pm 9 \mathrm{eV}$ and $a=1.17 \pm 0.10$.

This detector shows a much smaller LY compared to the one based on $\mathrm{Na}_{2} \mathrm{~W}_{2} \mathrm{O}_{7}: \mathrm{LY}_{\beta / \gamma}=1.61 \pm 0.01 \mathrm{keV} / \mathrm{MeV}$ and $\mathrm{LY}_{\alpha}=0.25 \pm 0.02 \mathrm{keV} / \mathrm{MeV}$. Due to the poor light yield, it is not possible to disentangle nuclear recoils from the band of $\beta / \gamma$ and thus to estimate their light emission.

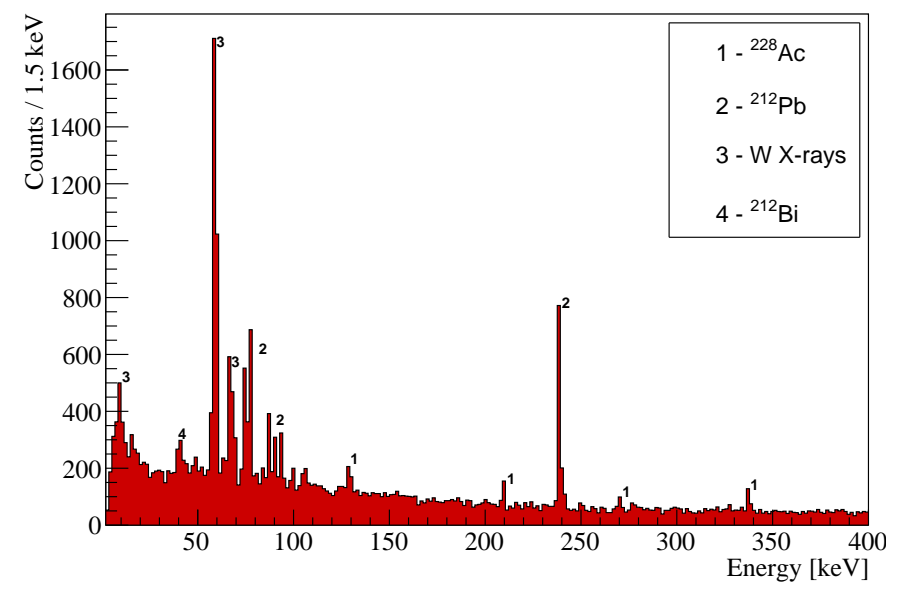

Figure 6: Energy spectrum of the $\mathrm{Na}_{2} \mathrm{Mo}_{2} \mathrm{O}_{7}$ crystal exposed to the thoriated tungsten wire.

We highlight that the presence of ${ }^{100} \mathrm{Mo}$, a golden candidate for neutrino-less double beta decay searches, makes this compound very interesting also for a multi-physics experimental approach. Indeed, in the last years the community of double beta decay searches performed an intense R\&D activity devoted to the development of crystals containing ${ }^{100} \mathrm{Mo}$ [37, 38, 16, 39, 40].

We extract the energy resolution in the region of interest for this process $\left(Q_{\text {value }}=3034.40(17) \mathrm{keV}\right.$ [41] $)$, obtaining $\sigma\left({ }^{100} \mathrm{Mo}\right)=3.77 \pm 0.31 \mathrm{keV}$. This result is similar to those obtained with other promising compounds, proving the high potential of this crystal that, unlike $\mathrm{Li}_{2} \mathrm{MoO}_{4}$ selected for the CUPID experiment [17, 42], is not hygroscopic.

\section{Conclusion and perspectives}

In this paper, we presented the first bolometric measurement of two Na-containing crystals, $\mathrm{Na}_{2} \mathrm{Mo}_{2} \mathrm{O}_{7}$ and $\mathrm{Na}_{2} \mathrm{~W}_{2} \mathrm{O}_{7}$.
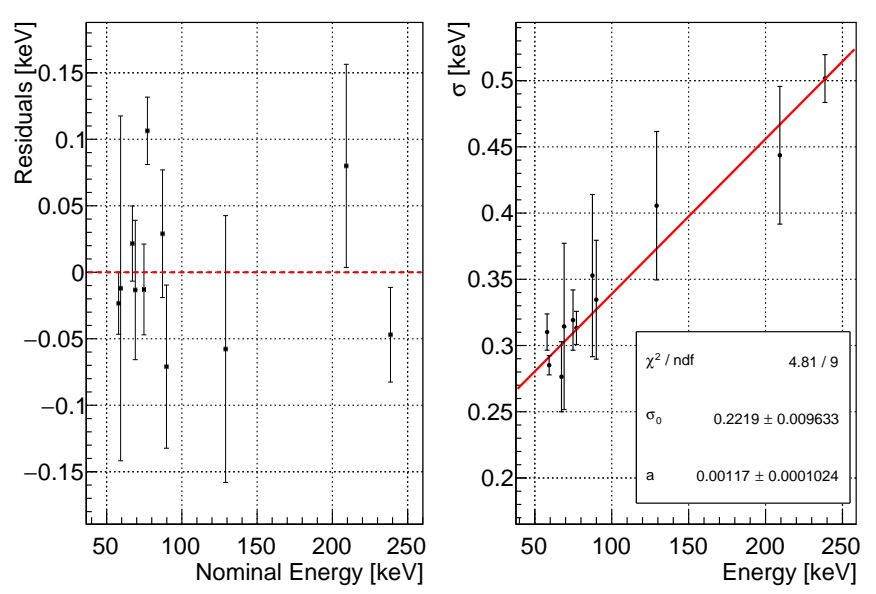

Figure 7: Left: Residuals of the energy calibration (nominal energy - reconstructed energy) as a function of the energy for $\mathrm{Na}_{2} \mathrm{Mo}_{2} \mathrm{O}_{7}$. Right: energy resolution as a function of the energy. We modeled the dependency on the energy using a linear function $\sigma(E)=\sigma_{0}+a E$.

Despite the low purity of some of the starting materials, the internal contamination measured with ICPMS are extremely low, suggesting a very small segregation factor of impurities in these compounds.

Both the crystals show a high signal response and a baseline energy resolution of the order of $\approx 200 \mathrm{eV}$ RMS. These preliminary results are very encouraging, as they would guarantee the $1 \mathrm{keV}$ energy threshold required to reach a sensitivity comparable to the one of DAMA/LIBRA.

The energy resolution of these prototypes increases linearly as a function of the energy but remains excellent for both the compounds. This feature is of particular interest for $\mathrm{Na}_{2} \mathrm{Mo}_{2} \mathrm{O}_{7}$, which could enable the simultaneous search of dark matter interactions and neutrino-less double beta decay of ${ }^{100}$ Mo. In the region of interest for this process, indeed, we measured $\sigma=(3.77 \pm 0.31) \mathrm{keV}$, which is compatible with other Mo-containing bolometers.

We made a preliminary study of the light yield, proving that both the crystals show satisfactory particle identification capabilities at high energies. The high pile-up rate in the light detector (due to the presence of the -very intense- thoriated tungsten wire) prevented a more detailed study of the light yield of nuclear recoils, on which we could just set upper limits.

This pilot experiment proves that both the compounds should be further studied for applications in rare events searches.

\section{Acknowledgements}

We thank the CUPID-0 and the CUORE collaborations for the overall support and for sharing their DAQ and software. We express our gratitude to LNGS for the generous hospitality and, in particular, we want to thank the LNGS mechanical workshop team E. Tatananni, A. Rotilio, A. Corsi, and B. Romualdi for continuous and constructive help and M. Guetti for his cryogenic expertise and his constant support. 


\section{References}

[1] P. A. R. Ade et al., "Planck 2015 results. XIII. Cosmological parameters," Astron. Astrophys., vol. 594, p. A13, 2016.

[2] R. Bernabei et al., "First model independent results from DAMA/LIBRAphase 2 ," Nucl. Phys. At. Energy, vol. 19, p. 307, 2019.

[3] S. C. Kim et al., "New Limits on Interactions between Weakly Interacting Massive Particles and Nucleons Obtained with CsI(Tl) Crystal Detectors," Phys. Rev. Lett., vol. 108, p. 181301, 2012.

[4] K. Fushimi et al., "Dark matter search project PICO-LON," J. Phys. Conf. Ser., vol. 718, no. 4, p. 042022, 2016.

[5] J. Amar et al., "First Results on Dark Matter Annual Modulation from the ANAIS-112 Experiment," Phys. Rev. Lett., vol. 123, no. 3, p. 031301, 2019.

[6] G. Adhikari et al., "Search for a Dark Matter-Induced Annual Modulation Signal in NaI(Tl) with the COSINE-100 Experiment," Phys. Rev. Lett., vol. 123 , no. 3, p. 031302, 2019

[7] M. Antonello et al., "The SABRE project and the SABRE Proof-ofPrinciple," Eur. Phys. J., vol. C79, no. 4, p. 363, 2019.

[8] G. Angloher et al., "Results from the first cryogenic NaI detector for the COSINUS project," JINST, vol. 12, no. 11, p. P11007, 2017.

[9] G. Angloher et al., "A CsI low temperature detector for dark matter search,” Astropart. Phys., vol. 84, pp. 70-77, 2016.

[10] G. Angloher et al., "The COSINUS project - perspectives of a NaI scintillating calorimeter for dark matter search," Eur. Phys. J., vol. C76, no. 8, p. 441, 2016.

[11] S. Pirro and P. Mauskopf, "Advances in Bolometer Technology for Fundamental Physics," Ann. Rev. Nucl. Part. Sci., vol. 67, pp. 161-181, 2017.

[12] C. Alduino et al., "First Results from CUORE: A Search for Lepton Number Violation via $0 v \beta \beta$ Decay of ${ }^{130}$ Te," Phys. Rev. Lett., vol. 120, no. 13, p. 132501, 2018.

[13] O. Azzolini et al., "Final result of CUPID-0 phase-I in the search for the ${ }^{82}$ Se Neutrinoless Double Beta Decay," Phys. Rev. Lett., 2019.

[14] O. Azzolini et al., "Search of the neutrino-less double beta decay of ${ }^{82} \mathrm{Se}$ into the excited states of ${ }^{82} \mathrm{Kr}$ with CUPID-0," Eur. Phys. J., vol. C78, no. 11, p. 888, 2018.

[15] O. Azzolini et al., "Analysis of cryogenic calorimeters with light and hea read-out for double beta decay searches," Eur. Phys. J., vol. C78, no. 9, p. 734, 2018.

[16] E. Armengaud et al., "Development of ${ }^{100}$ Mo-containing scintillating bolometers for a high-sensitivity neutrinoless double-beta decay search," Eur. Phys. J., vol. C77, no. 11, p. 785, 2017.

[17] E. Armengaud et al., "The CUPID-Mo experiment for neutrinoless double-beta decay: performance and prospects," Submitted to Eur. Phys. J. C, 2019.

[18] V. Alenkov et al., "First Results from the AMoRE-Pilot neutrinoless double beta decay experiment," Eur. Phys. J., vol. C79, 2019.

[19] A. H. Abdelhameed et al., "First results from the CRESST-III low-mass dark matter program, astro-ph:1904.00498," Phys. Rev. D, 2019.

[20] E. Armengaud et al., "Searches for electron interactions induced by new physics in the EDELWEISS-III Germanium bolometers," Phys. Rev., vol. D98, no. 8, p. 082004, 2018.

[21] R. Agnese et al., "First Dark Matter Constraints from a SuperCDMS Single-Charge Sensitive Detector," Phys. Rev. Lett., vol. 121, no. 5, p. 051301, 2018. [erratum: Phys. Rev. Lett.122,no.6,069901(2019)].

[22] J. W. Beeman et al., "Characterization of bolometric Light Detectors for rare event searches," JINST, vol. 8, p. P07021, 2013.

[23] A. V.V. et al., "Structural and electronic properties of $\mathrm{ZnWO}_{4}(010)$ cleaved surface," Cryst. Growth Des., vol. C78, no. 11, pp. 2479-2484, 2011.

[24] V. Ya.V. et al., "Low-gradient growth of oxide scintillator crystals," Scintillator materials. Engineering, devices, applications, Kharkov, ISMA, vol. 2011, no. 2011, pp. 119-180, 2011.

[25] S. D.A. et al., "Low temperature luminescence of $\mathrm{ZnMoO}_{4}$ single crystals grown by low temperature gradient Czochralski technique," Opt. Mater., vol. 34, pp. 1804-1810, 2012.

[26] Y. Borovlev, N. Ivannikova, V. Shlegel, Y. Vasiliev, and V. Gusev, "Progress in growth of large sized bgo crystals by the low-thermalgradient czochralski technique," Journal of Crystal Growth, vol. 229, no. 1, pp. $305-311,2001$. 1st Asian Conf. on Crystal Growth and Crystal Technology.
[27] T. Gavrilova, N. Ivannikova, V. Shlegel, V. Grigorieva, S. Solodovnikov, T. Bekker, and V. Atuchin, "Growth of $\mathrm{na}_{2} \mathrm{~W}_{2} \mathrm{O}_{7}$ single crystals as possible optical host material," in Physics and Technology of Nanostructured Materials II, vol. 213 of Solid State Phenomena, pp. 160-164, Trans Tech Publications Ltd, 32014.

[28] V. N. Shlegel et al., "Purification of molybdenum oxide, growth and characterization of medium size zinc molybdate crystals for the LUMINEU program," EPJ Web Conf., vol. 65, p. 03001, 2014.

[29] V. Grigorieva, N. Ivannikova, I. Ivanov, E. Makarov, I. Novoselov, and V. Shlegel, "Precursors preparation for growth of low-background scintillation crystals," AIP Conf. Proc., vol. 1921, no. 1, p. 080002, 2018.

[30] V. Grigorieva et al., " $\mathrm{Na}_{2} \mathrm{Mo}_{2} \mathrm{O}_{7}$ scintillating crystals: Growth, morphology and optical properties," Journal of Crystal Growth, vol. 507, pp. 3137, 2019.

[31] C. Arnaboldi et al., "The programmable front-end system for CUORICINO, an array of large-mass bolometers," IEEE Trans. Nucl. Sci., vol. 49, no. 5, pp. 2440-2447, 2002

[32] C. Arnaboldi et al., "The front-end readout for CUORICINO, an array of macro-bolometers and MIBETA, an array of mu-bolometers," Nucl. Instrum. Meth., vol. A520, pp. 578-580, 2004.

[33] S. Di Domizio, A. Branca, A. Caminata, L. Canonica, S. Copello, A. Giachero, E. Guardincerri, L. Marini, M. Pallavicini, and M. Vignati, "A data acquisition and control system for large mass bolometer arrays," JINST, vol. 13, no. 12, p. P12003, 2018.

[34] E. Gatti and P. F. Manfredi, "Processing the signals from solid state detectors in elementary particle physics," Riv. Nuovo Cimento, vol. 9, p. 1, 1986.

[35] G. Piperno, S. Pirro, and M. Vignati, "Optimizing the energy threshold of light detectors coupled to luminescent bolometers," JINST, vol. 6, p. P10005, 2011.

[36] V. I. Tretyak, "Semi-empirical calculation of quenching factors for scintillators: new results," EPJ Web Conf., vol. 65, p. 02002, 2014.

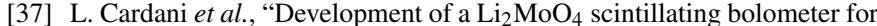
low background physics," JINST, vol. 8, p. P10002, 2013.

[38] J. W. Beeman et al., " $\mathrm{ZnMoO}_{4}$ : A Promising bolometer for neutrinoless double beta decay searches," Astropart. Phys., vol. 35, pp. 813-820, 2012.

[39] S. Nagorny, L. Pattavina, M. B. Kosmyna, B. P. Nazarenko, S. Nisi, L. Pagnanini, S. Pirro, K. Schffner, and A. N. Shekhovtsov, "arch $\mathrm{PbMoO}_{4}$ scintillating bolometer as detector to searches for the neutrinoless double beta decay of ${ }^{100}$ Mo," J. Phys. Conf. Ser., vol. 841, no. 1, p. 012025, 2017.

[40] L. Pattavina, S. Nagorny, S. Nisi, L. Pagnanini, G. Pessina, S. Pirro, C. Rusconi, K. Schaeffner, V. N. Shlegel, and V. N. Zhdankov, "Production and characterization of a $\mathrm{PbMoO}_{4}$ cryogenic detector from archeological Pb," Submitted to Eur. Phys. J. A, 2019.

[41] S. Rahaman et al., "Q value of the Mo-100 Double-Beta Decay," Phys. Lett., vol. B662, pp. 111-116, 2008.

[42] W. R. Armstrong et al., “CUPID pre-CDR, arXiv 1907.09376,” 2019. 